\title{
Structural Insights into the Anti-methicillin-resistant Staphylococcus aureus (MRSA) Activity of Ceftobiprole*
}

Received for publication, February 28, 2012, and in revised form, July 18, 2012 Published, JBC Papers in Press, July 19, 2012, DOI 10.1074/jbc.M112.355644

\author{
Andrew L. Lovering ${ }^{\ddagger 1,2}$, Michael C. Gretes ${ }^{\ddagger 1,3}$, Susan S. Safadi ${ }^{\ddagger}$, Franck Danel ${ }^{\S}$, Liza de Castro ${ }^{\ddagger}$, Malcolm G. P. Page ${ }^{\S}$, \\ and Natalie C. J. Strynadka ${ }^{\ddagger 4,5}$
}

From the ${ }^{\ddagger}$ Department of Biochemistry and Molecular Biology and the Center for Blood Research, University of British Columbia, Vancouver, British Columbia, Canada V6T $1 Z 3$ and ${ }^{\S}$ Basilea Pharmaceutica International, Ltd., 4058 Basel, Switzerland

\begin{abstract}
Background: Ceftobiprole is a $\beta$-lactam recently developed to treat methicillin-resistant Staphylococcus aureus (MRSA) by inhibiting its antibiotic resistance determinant PBP2a.

Results: The PBP2a-ceftobiprole complex reveals an extensive binding interface with two distinct inhibitor conformations. Conclusion: Ceftobiprole inhibits PBP2a via increased stabilization of the Michaelis complex followed by acylation.

Significance: We report the first structure of a resistant PBP inhibited by a competent anti-MRSA $\beta$-lactam.
\end{abstract}

Methicillin-resistant Staphylococcus aureus (MRSA) is an antibiotic-resistant strain of $S$. aureus afflicting hospitals and communities worldwide. Of greatest concern is its development of resistance to current last-line-of-defense antibiotics; new therapeutics are urgently needed to combat this pathogen. Ceftobiprole is a recently developed, latest generation cephalosporin and has been the first to show activity against MRSA by inhibiting essential peptidoglycan transpeptidases, including the $\boldsymbol{\beta}$-lactam resistance determinant PBP2a, from MRSA. Here we present the structure of the complex of ceftobiprole bound to PBP2a. This structure provides the first look at the molecular details of an effective $\boldsymbol{\beta}$-lactam-resistant PBP interaction, leading to new insights into the mechanism of ceftobiprole efficacy against MRSA.

Methicillin-resistant Staphylococcus aureus (MRSA) ${ }^{6}$ is a strain of $S$. aureus that has developed resistance to the $\beta$-lactam antibiotics, which include penicillins (e.g. methicillin, oxacillin, and dicloxacillin) and cephalosporins (1-3). Over the past dec-

* This work was supported by operating grants from the Canadian Institutes of Health Research and Howard Hughes Medical Institute and infrastructure grants from the Michael Smith Foundation of Health Research and the Canada Foundation for Innovation (to N. C. J. S.).

The atomic coordinates and structure factors (code 4DKI) have been deposited in the Protein Data Bank, Research Collaboratory for Structural Bioinformatics, Rutgers University, New Brunswick, NJ (http://www.rcsb.org/).

${ }^{1}$ Both authors contributed equally to this work.

${ }^{2}$ Supported by a New Investigator award from the Royal Society. Present address: School of Biosciences, University of Birmingham, Birmingham B15 2TT, UK.

${ }^{3}$ Present address: Dept. of Biochemistry and Biophysics, Oregon State University, Corvallis, OR 97331.

${ }^{4}$ Howard Hughes Medical Institute international research scholar, Michael Smith Foundation for Health Research Senior Scholar, and a Canada Research Tier 1 Chair in Antibiotic Discovery.

${ }^{5}$ To whom correspondence should be addressed: Dept. of Biochemistry and Molecular Biology and the Center for Blood Research, University of British Columbia, Life Sciences Center, 2350 Health Sciences Mall, Vancouver, BC, Canada V6T 1Z3. Tel.: 604-822-0789; Fax: 604-822-5227; E-mail: natalie@ byron.biochem.ubc.ca.

${ }^{6}$ The abbreviations used are: MRSA, methicillin-resistant Staphylococcus aureus; $\mathrm{MME}$, monomethyl ether; $\mathrm{PBP}$, penicillin-binding protein; $\mathrm{PDB}$, Protein Data Bank; r.m.s.d., root mean square deviation. ade, there has been a striking increase in the prevalence of hospital-acquired MRSA infections as well as an emergent epidemic of community-acquired MRSA infections (4). Most disconcerting is the development of resistance in $S$. aureus to current last-resort antibiotics including vancomycin (5) and linezolid (6). Ceftobiprole (see Fig. $1 a$ ) is among a new generation of cephalosporin $\beta$-lactams that combines activity against MRSA with broad spectrum activity against Gram-negative bacteria and other Gram-positive bacteria $(7,8)$. Ceftobiprole was the first $\beta$-lactam shown to possess anti-MRSA activity (and also the first antibiotic with potency against both MRSA and Pseudomonas aeruginosa); in addition to inhibiting the methicillin-susceptible PBPs of S. aureus (2), it remains able to target both Enterococcus faecalis and penicillin-resistant Streptococcus pneumoniae. Thus, ceftobiprole has a broad spectrum of activity encompassing several prominent pathogens. To elucidate the molecular details of the action of ceftobiprole against its target for inhibition, PBP2a, we present here the structure of PBP2a in complex with ceftobiprole.

The bacterial cell wall peptidoglycan is a complex structure composed of glycan strands cross-linked through peptide side chains. Transpeptidases belong to a group of enzymes known historically as penicillin-binding proteins (PBPs), which catalyze the formation of specific cross-links between the peptide side chains of neighboring glycan strands, a process essential to cell wall integrity $(9,10)$. The mechanism of $\beta$-lactam inhibition of this transpeptidation step in bacterial cell wall biosynthesis is well documented: $\beta$-lactams function specifically as substrate analogs of the D-Ala-D-Ala peptidoglycan side chain termini upon which a PBP acts, forming a long-lived inhibitory covalent acyl-enzyme complex with the nucleophilic serine within the enzyme active site. The region of the active site that normally accommodates the deacylating acceptor moiety of an adjacent peptidoglycan strand or a potential hydrolyzing water molecule remains occupied by the fused ring system of the $\beta$-lactam; hence, the antibiotic-bound PBP cannot undergo its usual subsequent deacylation. With the enzyme now inactivated, the consequent loss of cell wall cross-linking ultimately leads to cell lysis and death (11-13). This can be described by Reaction 1 . 


\section{Anti-MRSA Activity of Ceftobiprole}

$$
\mathrm{PBP}+\beta \text {-lactam } \stackrel{K_{d}}{\longleftrightarrow} \mathrm{PBP} \cdot \beta \text {-lactam } \underset{[\text { Michaelis complex }]}{\stackrel{k_{2}}{\longrightarrow}}
$$

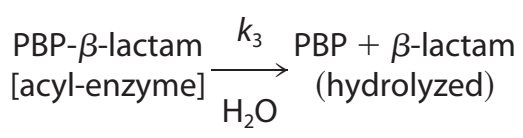

\section{REACTION 1}

Here, the $\beta$-lactam initially binds a PBP noncovalently (with dissociation constant $K_{d}$ ) to form the Michaelis complex. The PBP serine nucleophile then attacks the $\beta$-lactam peptide bond to create the covalent acyl-enzyme complex (at the rate indicated by the constant $k_{2}$ ). Nucleophilic attack by water would regenerate the enzyme; however, $\beta$-lactam antibiotics are hydrolyzed extremely slowly by PBPs (reflected by low $k_{3}$ values $(14,15))$ so in effect the acyl-PBP is now irreversibly inhibited. PBP2a is believed to resist inactivation via a very slow acylation step (its $k_{2}$ is 3 orders of magnitude lower than $\beta$-lactam sensitive PBPs (16)). Meanwhile, PBP2a exhibits $\beta$-lactam binding affinity $\left(K_{d}\right)$ and poor deacylation efficiency $\left(k_{3}\right)$ values comparable with sensitive PBPs $(17,18)$. Thus, a $\beta$-lactam with improved binding affinity (decreased $K_{d}$ ) would feature increased inhibition resulting from greater overall acylation efficiency $\left(k_{2} / K_{d}\right)$; in other words, the low probability that the Michaelis complex will yield an acylation event would be compensated for by increasing the frequency with which the Michaelis complex forms.

MRSA evades inhibition by $\beta$-lactams through acquisition of an exogenous, $\beta$-lactam-resistant PBP, PBP2a (also referred to as PBP2'), encoded by the mecA gene (19-23). Crystal structures of both the methicillin-susceptible PBP2 (24) and methicillin-resistant PBP2a (16) have provided the molecular details describing the mechanism of $\beta$-lactam resistance achieved by PBP2a. Most significantly, they show a structural rearrangement around the active site serine (Ser-403) which is located in a narrow, extended cleft in the resistant PBP2a (see Fig. 2). This rearrangement, although still allowing for initial binding of the antibiotic, causes displacement and misalignment of the $\beta$-lactam with respect to the serine nucleophile. Therefore, a productive $\beta$-lactam arrangement in the PBP2a active site requires a significant conformational change to occur, presumably making initiation of the acylation reaction less frequent (decreased $k_{2}$ ). It is postulated that any anti-MRSA $\beta$-lactam would owe its effectiveness to increased hydrophobic interaction with PBP2a, increasing the frequency of formation of the Michaelis complex and compensating for the energetic cost of rearrangement (16).

To test these existing hypotheses about anti-MRSA $\beta$-lactam drug design, we obtained the first structure of PBP2a in complex with ceftobiprole (deposited in the Protein Data Bank (PDB) with accession code 4DKI). Ceftobiprole is a pyrrolidinone cephalosporin that has an oxyimino aminothiadiazolyl substituent (R1 group) linked to the 7-amino group of the cephalosporin nucleus, conferring stability to hydrolysis by many $\beta$-lactamases. A vinylpyrrolidinone moiety ( 2 group) located at position 3 facilitates interaction with the narrow groove of the PBP2a active site and favors the acylation of PBP2a (Fig. 1a).
TABLE 1

\begin{tabular}{|c|c|}
\hline \multicolumn{2}{|l|}{ Crystal parameters $^{a}$} \\
\hline Space group & $\mathrm{P} 2_{1} 2_{1} 2_{1}$ \\
\hline Cell dimensions: $a \times b \times c, \AA$ & $80.8 \times 103.5 \times 186.5$ \\
\hline Resolution, $\AA$ & $2.9(3.06-2.9)$ \\
\hline Wavelength, $\AA$ & 1.11588 \\
\hline No. reflections & 106,806 \\
\hline No. unique reflections & 32,591 \\
\hline Average redundancy & $3.3(2.8)$ \\
\hline Completeness, \% & $91.9(63.5)$ \\
\hline$I / \sigma I$ & $15.9(3.3)$ \\
\hline$R_{\mathrm{sym}}{ }^{b}$ & $0.07(0.248)$ \\
\hline \multicolumn{2}{|l|}{ Refinement statistics $^{c}$} \\
\hline $\begin{array}{l}\text { Atoms in the crystallographic } \\
\text { asymmetric unit }\end{array}$ & $\begin{array}{l}1275 \text { observable amino acid residues, } 2 \\
\text { ceftobiprole molecules, } 39 \mathrm{H}_{2} \mathrm{O}, 7 \\
\mathrm{Cd}^{2+}, 4 \mathrm{Cl}^{-}, 4 \text { bicarbonate ions. }\end{array}$ \\
\hline$R_{\text {work }} / R_{\text {free }}$ & $0.173 / 0.237$ \\
\hline r.m.s.d. bond lengths, $\AA$ & 0.010 \\
\hline r.m.s.d. bond angles, degrees & 1.31 \\
\hline
\end{tabular}

Data collection and refinement statistics

\section{EXPERIMENTAL PROCEDURES}

Protein Expression, Purification, and Crystallization-S. aureus PBP2a was prepared as described previously (16). Briefly, a construct expressing residues 23-668 (with the mutation $\mathrm{Y} 23 \mathrm{M})$ produced soluble recombinant protein in Escherichia coli, lacking the $\mathrm{N}$-terminal transmembrane sequence. PBP2a was purified to near homogeneity through sequential Q-Sepharose, CM-Sepharose, hydroxyapatite, and Sephacryl100 chromatography steps. The protein was concentrated to 20 $\mathrm{mg} / \mathrm{ml}$ in a final buffer of $5 \mathrm{~mm} \mathrm{NaHCO}_{3}, \mathrm{pH} 8.0,150 \mathrm{~mm} \mathrm{NaCl}$. Crystals of the orthorhombic form of PBP2a were grown using $1 \mathrm{ml}$ of protein crystal stock mixed with $1 \mathrm{ml}$ of reservoir solution (100 mm Hepes, pH 7.0, $0.88 \mathrm{~m} \mathrm{NaCl}, 20 \%$ (v/v) PEG 500 MME, and $16 \mathrm{mM} \mathrm{CdCl}_{2}$ ) in a typical sitting drop vapor-diffusion experiment.

Derivatization, Data Collection, and Structure DeterminationCeftobiprole (Basilea Pharmaceutica) was solubilized in dimethyl sulfoxide (with $0.2 \% \mathrm{v} / \mathrm{v}$ TFA, agitated for $20 \mathrm{~min}$ at room temperature) to give a stock solution of $20 \mathrm{~mm}$. This was diluted 1:200 into PBP2a cryoprotection solution (100 mM Hepes, pH 7.0, $1 \mathrm{~m} \mathrm{NaCl}$,28\% (v/v) PEG $550 \mathrm{MME}$, and 16 $\mathrm{mM} \mathrm{CdCl} 2$ ). This derivatization solution was added directly to the crystallization drop (in three $1-\mu$ l increments, over a time course of $35 \mathrm{~min}$ ) and left overnight to promote acylation. Acylated PBP2a crystals were plunge-cooled to $100 \mathrm{~K}$ and screened for diffraction quality. Data were collected at Beamline 8.3.1 of the Advanced Light Source (Berkeley, CA), using $\mathrm{x}$-rays of $1.116 \AA$ wavelength. Full statistics are reported in Table 1.

Data Processing and Refinement-Data were processed using MOSFLM, scaled with SCALA, and data file manipulations performed using the CCP4 suite of programs (25). Due to the isomorphous nature of the crystals with those previously reported (16), the coordinates of the PBP2a nitrocefin acyl-enzyme (PDB code 1MWS) were used to generate initial phases. The possibility of model bias arising from the inclusion of the nitrocefin adduct in the initial phase estimates was negated through deletion of the nitrocefin adduct from the model and the usage of the Prime and Switch procedure in RESOLVE (26) to provide maps for the modeling of the ceftobiprole moiety. 
a

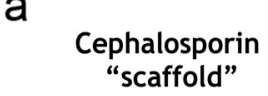

"ephalosporin

"scaffold"

Ceftobiprole<smiles>C/C(=N\O)C(=O)C(=O)NC1C(=O)N2C(C(=O)O)=C(/C=C3\CCN(C4CCNC4)C3=O)CSC12</smiles>

Nitrocefin<smiles>O=C(Cc1ccsc1)NC1C(=O)N2C(C(=O)O)=C(/C=C/c3ccc([N+](=O)[O-])cc3[N+](=O)[O-])CSC12</smiles>

Methicillin<smiles>[R]NC1C(=O)N2C(C(=O)O)=C([R2])CS[C@H]12</smiles><smiles>COc1cccc(OC)c1C(=O)NC1C(=O)N2C1SC(C)(C)C2C(=O)O</smiles>
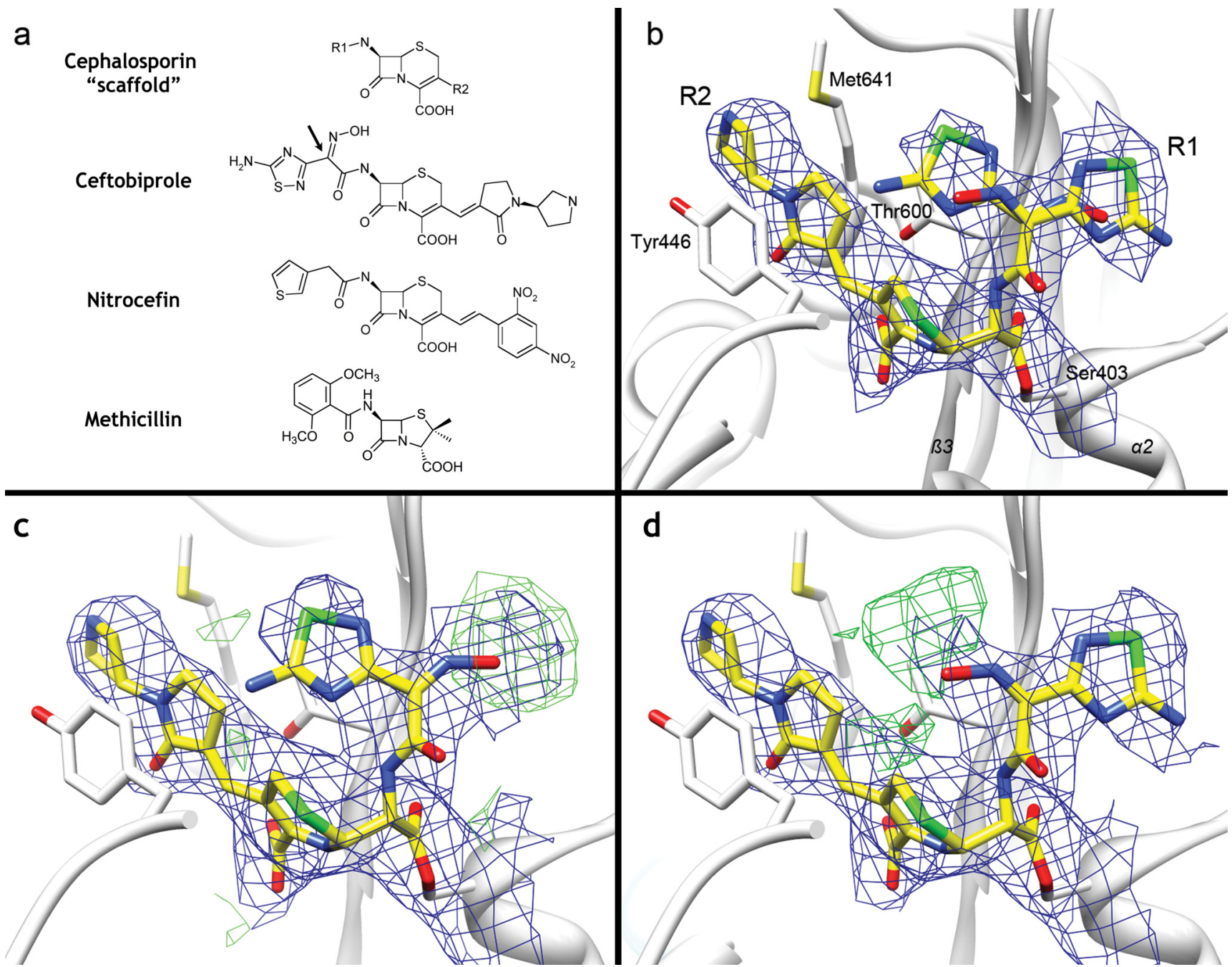

$$
\text { (20) }
$$
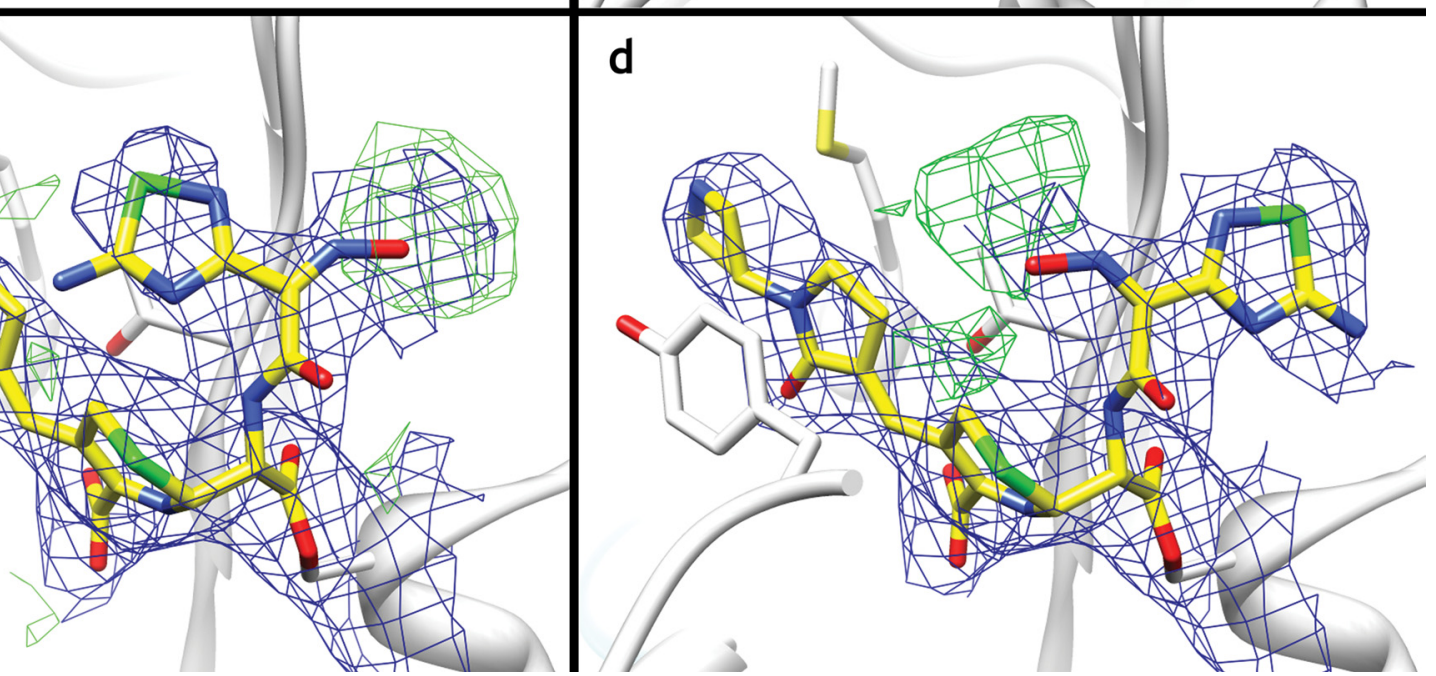

FIGURE 1. Chemical structures of MRSA-active and -inactive cephalosporins along with electron density maps and models of ceftobiprole covalently bound to Ser-403 at the PBP2a active site. $a$, general chemical structure of the cephalosporin antibiotics, indicating the positions of variable R1 and R2 groups and chemical structures of ceftobiprole, nitrocefin, and methicillin. $b$, final model and $2 F_{o}-F_{c}$ map contoured at $1 \sigma$. The ceftobiprole R1 group is shown in both of its proposed conformations A and B, with all atoms equivalent up to C7[C2] (indicated by an arrow in panel $a$ ). $C$, results of test refinement with ceftobiprole in the A conformation, with $2 F_{o}-F_{c}(b / u e, 1 \sigma)$ and $F_{o}-F_{c}(g r e e n, 3 \sigma)$ maps shown. The $F_{o}-F_{c}$ difference density is continuous with the modeled and unmodeled $2 F_{o}-F_{c}$ electron density, suggesting either multiple conformations or chemical modification of the terminal heterocycle of R1. With no evidence for the latter, and precedent for multiple R1 orientations among other transpeptidase structures, the modeling of two conformations was chosen $d$, as in $c$, with ceftobiprole modeled and test refined in the B conformation. Ceftobiprole is colored by CMYK convention ( $\mathrm{C}$, yellow; $\mathrm{N}$, blue; $\mathrm{O}$, red; $\mathrm{S}$, green), and $\sigma$ represents the root mean square electron density in the crystallographic unit cell.

Initial geometric restraints for ceftobiprole were generated using the PRODRG server (27). For the initial refinement stages, CNS was used (28), with later stages using TLS in REFMAC (29). Due to the moderate resolution of the data, refinement utilized NCS restraints for residues 27-402 and 404668, with the acyl-Ser-403 excluded. Final refinement and map improvement were completed using TLS in BUSTER-TNT (30). PRODRG-generated ceftobiprole bond length and angle restraints used in the final refinement were manually adjusted using recently published small molecule bond lengths and angles (31); bond lengths and angles for its aminothiadiazolyl moiety were derived from the crystal structure of the analogous five-membered ring of methyl (Z)-(5-amino-1,2,4-thiadiazol3-yl)-((fluoromethoxy)imino) acetate found in the Cambridge Structural Database (32). The final model has an $R_{\text {work }} / R_{\text {free }}$ of $0.173 / 0.237$ and is of good stereochemical quality (Table 1). Although the difference between $R_{\text {work }}$ and $R_{\text {free }}$ may seem somewhat large (0.064), it seems to us reasonable given the resolution of the data; it is also consistent with previously reported ligand-bound structures of PBP2a (PDB code, $R_{\text {work }} /$ $R_{\text {free }}$ difference as follows: 1MWU, 0.061; 1MWT, 0.062; $1 M W R, 0.054)$. Figures were generated using PyMOL (33) and UCSF Chimera (34).

Differential Scanning Calorimetry-Thermal stability of PBP2a (isolated as above) under various conditions was investigated by differential scanning calorimetry using the VP-DSC microcalorimeter (MicroCal GE Healthcare). Solutions of 10 $\mu \mathrm{M}$ PBP2a in $20 \mathrm{~mm}$ Tris, adjusted to $\mathrm{pH} 7.5$ with $\mathrm{H}_{2} \mathrm{SO}_{4}, 1 \mathrm{M}$ $\mathrm{NaCl}$ with and without $\beta$-lactam were used as samples. All solutions were thoroughly degassed before use, and the reference cell in each experiment was filled with an aliquot of buffer against which the protein solution had previously been dialyzed overnight. All samples were scanned from 30 to $70{ }^{\circ} \mathrm{C}$, at a rate of $1^{\circ} \mathrm{C} / \mathrm{min}$. Data were base-line-corrected, smoothed using a 


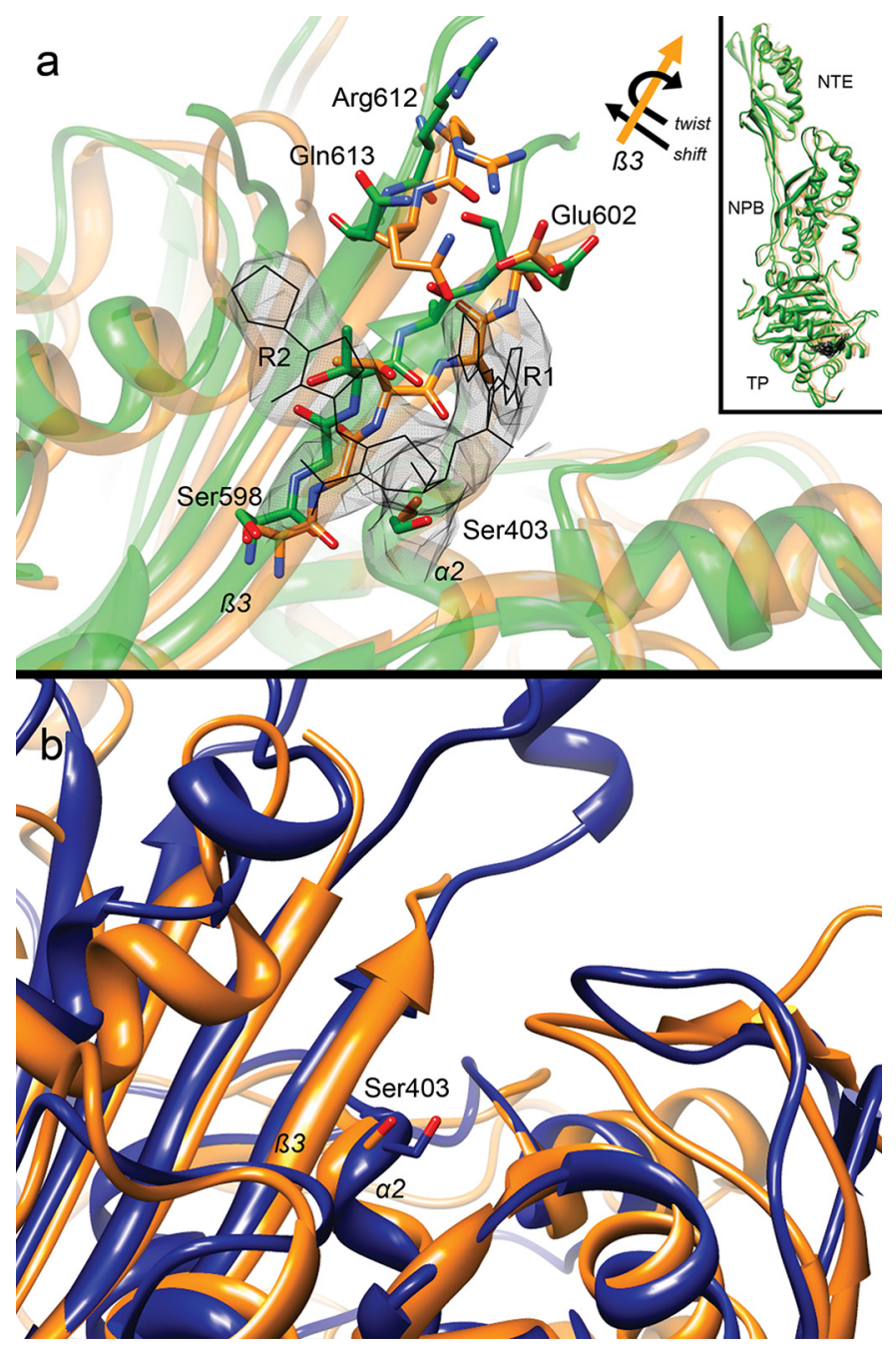

FIGURE 2. Structural comparisons demonstrating PBP2a structural rearrangements upon binding of ceftobiprole and the importance of serine nucleophile exposure. $a, \mathrm{PBP} 2 \mathrm{a}$ apo structure (orange) compared with PBP2a in complex with ceftobiprole (enzyme in green, ceftobiprole atomic bonds in black, and electron density contoured at $1 \sigma$ in gray). Secondary structure is shown as transparent ribbons, with backbone and side chain atoms overlaid in solid color. Upon binding, structural rearrangements must take place to accommodate ceftobiprole. These include a shift (toward the left in the figure) of the $\beta$-sheet, most notably at strand $\beta 3$, as well as a clockwise twist (relative to the direction of $\beta 3$ ) of the residue backbone and side chain atoms. The arrow diagram illustrates these rearrangements, and the inset depicts the whole PBP2a molecule with the active site indicated by the inhibitor (black). PBP2a domains are shown as follows: $\mathrm{N}$-terminal extension (NTE), non-penicillin-binding domain (NPB), and transpeptidase domain (TP). $b$, methicillin-susceptible endogenous PBP2 (blue) superimposed on the resistant PBP2a (orange). The O $\gamma$ atom of the PBP2 serine nucleophile (Ser398 ) is $2.0 \AA$ distant from that of PBP2a Ser-403 and so is far more exposed at the opening of the active site cleft, presumably contributing to its greater availability for acylation in vivo.

Savitsky-Golay 9-point smoothing algorithm, and analyzed using Origin Scientific plotting software.

\section{RESULTS}

PBP2a-Ceftobiprole Crystal Structure-The enzyme-inhibitor complex captured in our $\mathrm{x}$-ray structure shows clear electron density connecting the nucleophile Ser-403 with ceftobiprole, indicating the presence of an acyl-enzyme species. This is consistent with the reaction mechanism typical for $\beta$-lactams. Importantly, this observation indicates that ceftobiprole

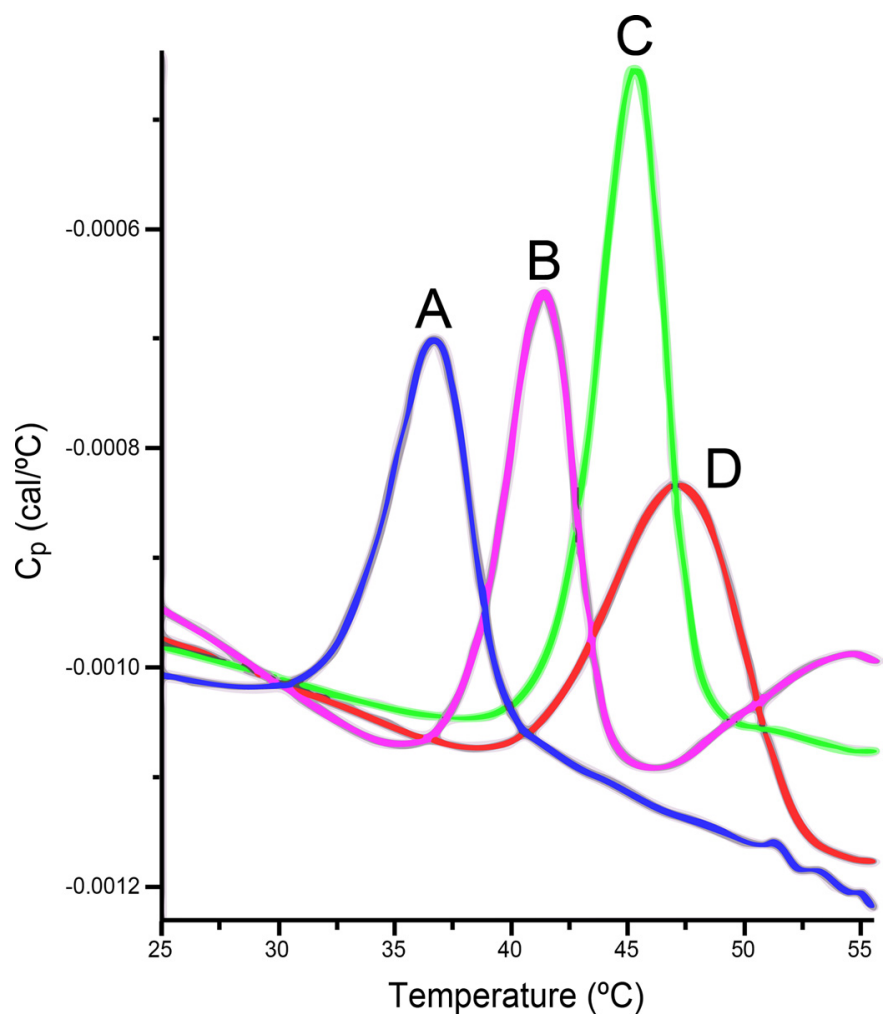

FIGURE 3. Differential scanning calorimetry traces for PBP2a and acylenzyme complexes formed with $\boldsymbol{\beta}$-lactams. $A$ (blue), heat capacity change in the presence of $1 \mathrm{~mm}$ imipenem. $B$ (magenta), heat capacity change in the presence of $100 \mu \mathrm{m}$ benzylpenicillin. C (green), heat capacity change observed for native protein. $D($ red), heat capacity change in the presence of $10 \mu \mathrm{m}$ ceftobiprole. Each $\beta$-lactam was also present in the reference cell, and traces in the absence of protein showed only a steady decline over the temperature range studied (data not shown).

behaves largely like a traditional $\beta$-lactam and does not derive its activity from an entirely novel mechanism.

Despite the moderate resolution of the diffraction data, it is clear that in monomer A of PBP2a the R1 substituent in the acyl-enzyme complex occupies two positions, denoted conformation $\mathrm{A}$, in which the $\mathrm{R} 1$ group is oriented toward the $\mathrm{R} 2$ group and residue Thr- 600 of PBP2a, and conformation B, in which the R1 group is oriented away from the R2 group and toward Ser-403 (Fig. 1, $b-d$ ).

Comparison of the enzyme-inhibitor complex structure with the previously published apo structure of PBP2a (PDB code 1VQQ) allows insight into the conformational changes required to accommodate ceftobiprole binding (Fig. 2a) (16). Upon acylation of PBP2a with ceftobiprole, a twisting of the central $\beta$-sheet, most notably at strand $\beta 3$, alters the position and orientation of Ser-598, Gly-599, and Thr-600 to avoid steric clashes with the reacted $\beta$-lactam. These backbone (and side chain $\mathrm{C}_{\beta}$ ) atoms twist, with a backbone-plus- $\mathrm{C}_{\beta}$ r.m.s.d. $\sim 1.2$ $\AA$. The neighboring Ala-601 and Glu-602 residues reposition themselves in response to the $\mathrm{R} 1$ substituent (all-atom r.m.s.d. $\sim 1.5 \AA$ ), as do residues Arg-612 and Gln-613 (all-atom r.m.s.d. $\sim 2.9 \AA$; for comparison, $625 \mathrm{C}_{\alpha}$ pairs of PBP2a upon acylation vary with r.m.s.d. $\sim 0.5 \AA$ ). The conformational change also involves movement of the $\mathrm{N}$ terminus of helix $\alpha 2$ such that Ser- 403 becomes more exposed and therefore better positioned to react (backbone-plus- $\mathrm{C}_{\beta}$, residues 403 and 404 have a 


\section{Anti-MRSA Activity of Ceftobiprole}

TABLE 2

Thermal stability determined for PBP2a upon acylation

\begin{tabular}{|c|c|c|c|c|c|}
\hline Compound & $T_{m}$ & $\Delta T_{m}$ & Peak height & Peak width at half-height & Total heat capacity \\
\hline & ${ }^{\circ} \mathrm{C}$ & ${ }^{\circ} \mathrm{C}$ & $\operatorname{mcal} /{ }^{\circ} \mathrm{C}$ & ${ }^{\circ} \mathrm{C}$ & mcal \\
\hline None & 45.8 & & 6.00 & 3.75 & 26.25 \\
\hline Ceftobiprole & 47.5 & +1.7 & 3.04 & 6.88 & 23.32 \\
\hline Cephalothin & 45.3 & -0.5 & 3.83 & 4.03 & 24.95 \\
\hline Nitrocefin & 45.2 & -0.6 & 3.95 & 3.83 & 23.12 \\
\hline Piperacillin & 43.4 & -2.4 & 4.54 & 3.80 & 20.95 \\
\hline Benzylpenicillin & 41.3 & -4.5 & 4.48 & 3.75 & 19.31 \\
\hline Imipenem & 36.5 & -9.3 & 3.63 & 4.17 & 18.70 \\
\hline
\end{tabular}

r.m.s.d. $\sim 0.9 \AA$ ). This is an important feature because serine nucleophile accessibility appears to be a major factor in determining whether acylation will occur as illustrated by the placement of Ser-403 deeper within the PBP2a active site cleft than the equivalent nucleophile in the methicillin-susceptible PBP2 (PDB code 2OLU; Fig. 2B; 16, 24). Apart from such reorganization events, the opposing side of the active site cleft evidently responds to acylation largely via rigid-body movement of regions $421-475$ (r.m.s.d. of 220 backbone-plus- $C_{\beta}$ atoms $\sim 0.9$ $\AA$ ) and 501-526 (r.m.s.d. of 100 backbone-plus- $C_{\beta}$ r.m.s.d. $\sim 1.1 \AA$ ).

Differential Scanning Calorimetry - In addition to our x-ray structure, thermal stability of PBP2a apo enzyme and six different acyl-enzyme complexes were compared using differential scanning calorimetry (Fig. 3). Of six $\beta$-lactams tested, only ceftobiprole was observed to induce an increase in the thermal stability of the complex formed with PBP2a (Table 2). The apo enzyme had a thermal transition $\left(T_{m}\right)$ at $45.8^{\circ} \mathrm{C}$ which was increased to $47.5^{\circ} \mathrm{C}$ with ceftobiprole. The cephalosporins cephalothin and nitrocefin each reproducibly caused a small $\left(0.5-0.6^{\circ} \mathrm{C}\right)$ shift to a lower temperature while also causing a decrease in the peak height and inducing a broadening of the transition. Piperacillin and benzylpenicillin produced larger shifts $\left(2-4{ }^{\circ} \mathrm{C}\right)$ toward lower $T_{m}$, and imipenem produced a profound decrease in the thermal stability $\left(T_{m}\right)$ of the protein, lowering the $T_{m}$ by $>9{ }^{\circ} \mathrm{C}$.

\section{DISCUSSION}

The ceftobibprole-PBP2a structure represents the first transpeptidase complex with a $\beta$-lactam containing an oxyimino aminothiadiazolyl R1 group captured in both A and B conformations in the same structure (each conformation has been observed individually in studies involving the similar R1 group of cefotaxime, but with different enzymes (35-37)). In general, it is observed that the R1 substituents tend toward conformation A in low molecular weight transpeptidases (monofunctional PBPs) and conformation B in the high molecular weight enzymes (multimodular PBPs).

We propose that movement in the outermost part of the active site region allows ceftobiprole to bind to the narrow cleft via hydrophobic interaction with the extended R2 group. Electron density is clear for the Tyr-446 side chain encompassed within this region and the ceftobiprole R2 group, whereas enzyme acylation has accompanied a disordering of the Met641 region. Met-641, Thr-600, and Tyr-446 sandwich the rings of the R2 substituent (forming favorable pi-pi stacking interactions); however, no hydrogen bonding occurs involving the $\mathrm{CO}$ or NH groups of the ceftobiprole R2 moiety. The hydrophobic nature of the R2 group was initially proposed to allow a nonspecific mode of interaction with PBP2a, which would allow for the $\beta$-lactam to be repositioned to accommodate any rearrangements near the Ser-403 nucleophile during the transition from the Michaelis complex to the acyl-PBP intermediate. The planarity and hydrophobicity of R2 indeed seem essential to allow the $\beta$-lactam extended access to the narrow active site groove; these properties mediate a close fit with Tyr-446, Met641, and Thr-600 and correspond well with the features of the pentaglycine cross-bridge, characteristic for $S$. aureus, which links together peptide side chains from neighboring glycan strands. Additionally, the clustering of anti-MRSA $\beta$-lactam structures around cephalosporins (and some carbapenems but not generally penams) supports the importance of this planar interaction (16). An increased number of van der Waals contacts with the R2 substituent of cephalosporins, which is not seen with penicillin nor with methicillin, is proposed to increase the binding affinity and result in the increased overall acylation rate observed for cephalosporins such as nitrocefin over that of penicillin $\mathrm{G}$ (18). The binding event is also associated with a rearrangement of the elements on the opposing side of the active site cleft, most notably at strand $\beta 3$ and helix $\alpha 2$. Because Thr-600 is located in strand $\beta 3$, contacts R2, and also forms part of the oxyanion hole, it appears to play a pivotal role in linking acylation to substrate binding in the course of $\beta 3$ strand rearrangements. The role of the $\beta$-lactam in facilitating changes at helix $\alpha 2$ is less obvious, although movement of Ser403 into the acylation position seems only possible via avoidance of steric clashes with helix $\alpha 2$ residue Lys-406 and strand $\beta 3$ upon formation of the Michaelis complex. Ring opening of the lactam forces the R1 and R2 groups into close proximity, an interaction well tolerated in the A conformation of ceftobiprole, but strikingly detrimental in bulkier compounds like methicillin, consistent with $\mathrm{MIC}_{90}$ values of $\leq 4 \mathrm{mg} / \mathrm{liter}$ for ceftobiprole compared with $1600 \mathrm{mg} /$ liter for methicillin (7, 39). Without information on the Michaelis complex with an unreacted $\beta$-lactam, it is difficult to identify roles for the R 1 and R2 groups in directly facilitating these conformational changes, because only the end point of the acylation reaction is observed.

Ceftobiprole binding raises the thermal transition of PBP2a by $\sim 2{ }^{\circ} \mathrm{C}$. Such increased thermal stability upon ligand binding is usually associated with the formation of additional interactions between the protein and ligand, which act to stabilize the complex. In contrast, the large decrease in thermal stability of PBP2a induced by imipenem, piperacillin, and benzylpenicillin (and to a lesser extent cephalothin and nitrocefin) suggests that the acyl-enzyme complexes formed with these $\beta$-lactams lose 


\section{Anti-MRSA Activity of Ceftobiprole}

stabilizing interactions during complex formation that are not compensated for by additional interactions with the inhibitor.

Changes in thermal stability of the acyl-enzyme complexes may influence kinetic measurements. For example, at $37^{\circ} \mathrm{C}(\mathrm{a}$ temperature often chosen for investigation of these kinetics (18)), the native protein is at the beginning of the unfolding transition and can be expected to be unstable under these conditions, whereas the imipenem complex $\left(T_{m} 36.5^{\circ} \mathrm{C}\right)$ would be at its thermal transition midpoint. Even at $25^{\circ} \mathrm{C}$, one would expect significant denaturation to occur if the measurements extend over hours (40). This suggests two possible modes of PBP2a inhibition: destabilization (e.g. by imipenem) or irreversible inhibition by stabilization of the Michaelis complex followed by acylation (e.g. by ceftobiprole).

The effectiveness of ceftobiprole is attributed to a combination of increased rate of acylation, higher intrinsic affinity, and a lower deacylation rate, all leading to greater inhibition of the active site of the transpeptidase (8). The differences between the resistant and susceptible PBPs in S. aureus result from altered positioning of the serine nucleophile, yielding large differences in acylation rates $(41,42)$. Ceftobiprole effectively acylates and inhibits PBP2a and displays potent inhibition against other PBPs (e.g. PBP2x in penicillin-resistant S. pneumoniae and PBP3 in E. coli) (43-45). Importantly, the potential for the development of resistance against ceftobiprole appears to be low, and it has been shown to be stable against hydrolysis by penicillinases produced by $S$. aureus as well as class A and C $\beta$-lactamases produced by certain Gram-negative bacteria (7, $38,46)$. In the presence of $\beta$-lactams, PBP2a can function as the sole PBP in cell wall biosynthesis. Therefore, it represents an excellent target for specific inhibitors against MRSA. The novel, broad spectrum antibiotic activity of ceftobiprole gives it great promise both in empiric therapy and in the design of other $\beta$-lactams to combat the growing resistance of several prominent pathogens.

Acknowledgments-We thank Gunnar Olovsson for maintenance of $x$-ray facilities and Daniel Lim for early efforts on the project and advice; Andy Karplus and Dale Tronrud at Oregon State University for helpful discussions and thoughtful suggestions regarding BusterTNT, which led to substantial improvements in the electron density map and refinement of the model; and Jarrod Johnson at University of Notre Dame for comments on $\beta$-lactam inhibitor conformation. We are grateful for beam time and assistance at the Advanced Light Source (ALS).

\section{REFERENCES}

1. Neu, H. C. (1992) The crisis in antibiotic resistance. Science 257, $1064-1073$

2. Lyon, B. R., and Skurray, R. (1987) Antimicrobial resistance of Staphylococcus aureus: genetic basis. Microbiol. Rev. 51, 88-134.

3. Barrett, F. F., McGehee, R. F., Jr., and Finland, M. (1968) Methicillinresistant Staphylococcus aureus at Boston City Hospital: bacteriologic and epidemiologic observations. New Engl. J. Med. 279, 441-448

4. Doshi, R. K., Patel, G., Mackay, R., and Wallach, F. (2009) Healthcareassociated infections: epidemiology, prevention, and therapy. Mt. Sinai J. Med. 76, 84-94

5. Sievert, D. M., Rudrik, J. T., Patel, J. B., McDonald, L. C., Wilkins, M. J., and Hageman, J. C. (2008) Vancomycin-resistant Staphylococcus aureus in the
United States, 2002-2006. Clin. Infect. Dis. 46, 668-674

6. Gales, A. C., Sader, H. S., Andrade, S. S., Lutz, L., Machado, A., and Barth, A. L. (2006) Emergence of linezolid-resistant Staphylococcus aureus during treatment of pulmonary infection in a patient with cystic fibrosis. Int. J. Antimicrob. Agents 27, 300-302

7. Hebeisen, P., Heinze-Krauss, I., Angehrn, P., Hohl, P., Page, M. G., and Then, R. L. (2001) In vitro and in vivo properties of Ro 63-9141, a novel broad-spectrum cephalosporin with activity against methicillin-resistant staphylococci. Antimicrob. Agents Chemother. 45, 825-836

8. Page, M. G. (2004) Cephalosporins in clinical development. Expert Opin. Investig. Drugs 13, 973-985

9. Ghuysen, J. M. (1994) Molecular structures of penicillin-binding proteins and $\beta$-lactamases. Trends Microbiol. 2, 372-380

10. Goffin, C., and Ghuysen, J. M. (1998) Multimodular penicillin-binding proteins: an enigmatic family of orthologs and paralogs. Microbiol. Mol. Biol. Rev. 62, 1079-1093

11. Kitano, K., and Tomasz, A. (1979) Triggering of autolytic cell wall degradation in Escherichia coli by $\beta$-lactam antibiotics. Antimicrob. Agents Chemother. 16, $838-848$

12. Shockman, G. D., Daneo-Moore, L., Kariyama, R., and Massidda, O. (1996) Bacterial walls, peptidoglycan hydrolases, autolysins, and autolysis. Microbial Drug Resistance 2, 95-98

13. Lewis, K. (2000) Programmed death in bacteria. Microbiol. Mol. Biol. Rev. 64, 503-514

14. Ghuysen, J. M., Frère, J. M., Leyh-Bouille, M., Nguyen-Distèche, M., and Coyette, J. (1986) Active-site-serine D-alanyl-D-alanine-cleaving-peptidase-catalysed acyl-transfer reactions: procedures for studying the penicillin-binding proteins of bacterial plasma membranes. Biochem. J. 235, $159-165$

15. Frère, J. M., Ghuysen, J. M., and Iwatsubo, M. (1975) Kinetics of interaction between the exocellular DD-carboxypeptidase-transpeptidase from Streptomyces R61 and $\beta$-lactam antibiotics: a choice of models. Eur. J. Biochem. 57, 343-351

16. Lim, D., and Strynadka, N. C. (2002) Structural basis for the $\beta$-lactam resistance of PBP2a from methicillin-resistant Staphylococcus aureus. Nat. Struct. Biol. 9, $870-876$

17. Lu, W. P., Sun, Y., Bauer, M. D., Paule, S., Koenigs, P. M., and Kraft, W. G. (1999) Penicillin-binding protein 2a from methicillin-resistant Staphylococcus aureus: kinetic characterization of its interactions with $\beta$-lactams using electrospray mass spectrometry. Biochemistry 38, 6537-6546

18. Graves-Woodward, K., and Pratt, R. F. (1998) Reaction of soluble penicillin-binding protein 2a of methicillin-resistant Staphylococcus aureus with $\beta$-lactams and acyclic substrates: kinetics in homogeneous solution. Biochem. J. 332, 755-761

19. Beck, W. D., Berger-Bächi, B., and Kayser, F. H. (1986) Additional DNA in methicillin-resistant Staphylococcus aureus and molecular cloning of mec-specific DNA. J. Bacteriol. 165, 373-378

20. Hartman, B. J., and Tomasz, A. (1984) Low-affinity penicillin-binding protein associated with $\beta$-lactam resistance in Staphylococcus aureus. J. Bacteriol. 158, 513-516

21. Matsuhashi, M., Song, M. D., Ishino, F., Wachi, M., Doi, M., Inoue, M., Ubukata, K., Yamashita, N., and Konno, M. (1986) Molecular cloning of the gene of a penicillin-binding protein supposed to cause high resistance to $\beta$-lactam antibiotics in Staphylococcus aureus. J. Bacteriol. 167, 975-980

22. Reynolds, P. E., and Brown, D. F. (1985) Penicillin-binding proteins of $\beta$-lactam-resistant strains of Staphylococcus aureus: effect of growth conditions. FEBS Lett. 192, $28-32$

23. Utsui, Y., and Yokota, T. (1985) Role of an altered penicillin-binding protein in methicillin- and cephem-resistant Staphylococcus aureus. Antimicrob. Agents Chemother. 28, 397-403

24. Lovering, A. L., de Castro, L. H., Lim, D., and Strynadka, N. C. (2007) Structural insight into the transglycosylation step of bacterial cell-wall biosynthesis. Science 315, 1402-1405

25. Collaborative Computational Project, Number 4 (1994) The CCP4 suite: programs for protein crystallography. Acta Crystallogr. D Biol. Crystallogr. 50, 760-763

26. Terwilliger, T. (2004) SOLVE and RESOLVE: automated structure solu- 


\section{Anti-MRSA Activity of Ceftobiprole}

tion, density modification and model building. J. Synchrotron Radiat. 11, $49-52$

27. Schüttelkopf, A. W., and van Aalten, D. M. (2004) PRODRG: a tool for high-throughput crystallography of protein-ligand complexes. Acta Crystallogr. D Biol. Crystallogr. 60, 1355-1363

28. Brünger, A. T., Adams, P. D., Clore, G. M., DeLano, W. L., Gros, P., Grosse-Kunstleve, R. W., Jiang, J. S., Kuszewski, J., Nilges, M., Pannu, N. S., Read, R. J., Rice, L. M., Simonson, T., and Warren, G. L. (1998) Crystallography \& NMR system: a new software suite for macromolecular structure determination. Acta Crystallogr. D Biol. Crystallogr. 54, 905-921

29. Murshudov, G. N., Vagin, A. A., and Dodson, E. J. (1997) Refinement of macromolecular structures by the maximum-likelihood method. Acta Crystallogr. D Biol. Crystallogr. 53, 240-255

30. Bricogne, G., Blanc, E., Brandl, M., Flensburg, C., Keller, P., Paciorek, W., Roversi, P., Smart, O. S., Vonrhein, C., and Womack, T. O. (2009) Global Phasing Ltd., Cambridge, UK

31. Pyykkö, P., and Atsumi, M. (2009) Molecular double-bond covalent radii for elements Li-E112. Chemistry 15, 12770-12779

32. Allen, F. H. (2002) The Cambridge Structural Database: a quarter of a million crystal structures and rising. Acta Crystallogr. B 58, 380-388

33. DeLano, W. L. (2010) The PyMOL Molecular Graphics System, version 1.3r1, Schrödinger, LLC, New York

34. Pettersen, E. F., Goddard, T. D., Huang, C. C., Couch, G. S., Greenblatt, D. M., Meng, E. C., and Ferrin, T. E. (2004) UCSF Chimera: a visualization system for exploratory research and analysis. J. Comput. Chem. 25, $1605-1612$

35. Contreras-Martel, C., Job, V., Di Guilmi, A. M., Vernet, T., Dideberg, O., and Dessen, A. (2006) Crystal structure of penicillin-binding protein 1a (PBP1a) reveals a mutational hotspot implicated in $\beta$-lactam resistance in Streptococcus pneumoniae. J. Mol. Biol. 355, 684-696

36. Macheboeuf, P., Di Guilmi, A. M., Job, V., Vernet, T., Dideberg, O., and Dessen, A. (2005) Active site restructuring regulates ligand recognition in class A penicillin-binding proteins. Proc. Natl. Acad. Sci. U.S.A. 102, $577-582$

37. Silvaggi, N. R., Josephine, H. R., Kuzin, A. P., Nagarajan, R., Pratt, R. F., and Kelly, J. A. (2005) Crystal structures of complexes between the R61 DDpeptidase and peptidoglycan-mimetic $\beta$-lactams: a non-covalent complex with a "perfect penicillin." J. Mol. Biol. 345, 521-533

38. Queenan, A. M., Shang, W., Kania, M., Page, M. G., and Bush, K. (2007) Interactions of ceftobiprole with $\beta$-lactamases from molecular classes $\mathrm{A}$ to D. Antimicrob. Agents Chemother. 51, 3089-3095

39. Sumita, Y., Fukasawa, M., Mitsuhashi, S., and Inoue, M. (1995) Binding affinities of $\beta$-lactam antibodies for penicillin-binding protein 2 ' in methicillin-resistant Staphylococcus aureus. J. Antimicrob. Chemother. 35, 473-481

40. Fuda, C., Suvorov, M., Vakulenko, S. B., and Mobashery, S. (2004) The basis for resistance to $\beta$-lactam antibiotics by penicillin-binding protein 2a of methicillin-resistant Staphylococcus aureus. J. Biol. Chem. 279, 40802-40806

41. Livermore, D. M. (2006) Can $\beta$-lactams be re-engineered to beat MRSA? Clin. Microbiol. Infect. 12, 11-16

42. Jones, M. E. (2007) Clin. Microbiol. Infect. 13, 17-24

43. Georgopapadakou, N. H. (1993) Penicillin-binding proteins and bacterial resistance to $\beta$-lactams. Antimicrob. Agents Chemother. 37, 2045-2053

44. Davies, T. A., Page, M. G., Shang, W., Andrew, T., Kania, M., and Bush, K. (2007) Binding of ceftobiprole and comparators to the penicillin-binding proteins of Escherichia coli, Pseudomonas aeruginosa, Staphylococcus aureus, and Streptococcus pneumoniae. Antimicrob. Agents Chemother. 51, 2621-2624

45. Chambers, H. F. (2003) Solving staphylococcal resistance to $\beta$-lactams. Trends Microbiol. 11, 145-148

46. Bogdanovich, T., Ednie, L. M., Shapiro, S., and Appelbaum, P. C. (2005) Antistaphylococcal activity of ceftobiprole, a new broad-spectrum cephalosporin. Antimicrob. Agents Chemother. 49, 4210-4219 